\title{
La construcción del entorno del Monasterio de San Lorenzo de El Escorial. Agua, territorio y paisaje
}

\author{
Building the surroundings of the Monastery of "San Lorenzo de El Escorial". \\ Water, territory and landscape \\ $\underline{\text { P. Chías }}^{(*)}$, T. Abad ${ }^{(*)}$
}

\section{RESUMEN}

Los estudios sobre el Monasterio del Escorial se han centrado tradicionalmente en las cualidades arquitectónicas, formales y estilísticas del conjunto, evitando abordar el complejo e imprescindible sistema de infraestructuras construido para su servicio y sin el que no hubiera podido desarrollar las actividades religiosas, formativas y cortesanas para las que fue proyectado. Los requisitos funcionales de un conjunto tan importante hicieron necesaria la construcción de un gran número de obras como el abastecimiento de agua y el saneamiento, pero también ingenios como los molinos o caminos para facilitar el acceso y asegurar su abastecimiento. La presente investigación aborda el estudio de las infraestructuras del agua, construidas ininterrumpidamente desde del siglo XVI para dar servicio en un primer momento al conjunto monacal, y después a la propia población de San Lorenzo. Esta compleja red ha aportado al territorio y al paisaje un formidable conjunto de hitos aún desconocidos en su mayoría ${ }^{1}$.

Palabras clave: Agua; territorio; patrimonio; paisaje; Monasterio de El Escorial.

\section{ABSTRACT}

Studies about the Monastery of El Escorial focused traditionally on the architectural, formal qualities of the monastic buildings, eluding other essential subjects as the infrastructures. It would have been impossible to develop the daily life wihout them. The needs of the different uses in such a complex ensemble needed a great set of buildings and infrastructures such as water supply and sewage, but also water mills and roads, to access and ensure the provisions. Our research deals with the study of all these hydraulic infrastructures, which were built continuously from the 16th century onwards, not only to serve the monastic buildings, but also the town of San Lorenzo. As complex net of civil works, it introduced in the territory and the landscape an essential set of landmarks, most of which still remain unknown.

Keywords: Water; territory; cultural heritage; landscape; Monastery of El Escorial.

(*) Escuela de Arquitectura - Universidad de Alcalá. Alcalá de Henares (España). Persona de contacto/Corresponding author: pilar.chias@uah.es (P. Chías)

Cómo citar este artículo/Citation: Chías, P., Abad, T. (2014). La construcción del entorno del Monasterio de San Lorenzo de El Escorial. Agua, territorio y paisaje. Informes de la Construcción, 66(536): e046, doi: http://dx.doi.org/10.3989/ic.14.027.

Licencia/License: Salvo indicación contraria, todos los contenidos de la edición electrónica de Informes de la Construcción se distribuyen bajo una licencia de uso y distribución Creative Commons Reconocimiento no Comercial 3.o. España (cc-by-nc).

\footnotetext{
${ }^{1}$ Los textos originales se han adaptado al español actual. Las principales abreviaturas utilizadas son: AME (Archivo del Monasterio de San Lorenzo de El Escorial, Madrid), BME (Biblioteca del Monasterio de San Lorenzo de El Escorial), AGS OyB (Archivo General de Simancas, Valladolid: Obras y Bosques), IVDJ (Instituto Valencia de Don Juan, Madrid), APR (Archivo del Palacio Real, Madrid).
} 


\section{INTRODUCCIÓN}

El entorno del Monasterio ha sido habitualmente contemplado desde un punto de vista esencialmente descriptivo, generalmente laudatorio y acrítico, como se aprecia tanto en las descripciones de los contemporáneos de la construcción de la fábrica -el Padre Sigüenza, Arfe, el doctor Almela, fray Juan de San Jerónimo- como en textos posteriores -fray Francisco de los Santos o Antonio Rotondo, entre otros-. Por su parte, visitantes y viajeros como Ponz, Gauthier o Unamuno proporcionaron unas visiones personales poco objetivas y muy mediatizadas por los prejuicios. También la mayoría de los estudios contemporáneos se ha centrado en los aspectos arquitectónicos del conjunto del Monasterio y de la población de San Lorenzo, relegando a un segundo término sus aspectos funcionales y su relación con el entorno.

Excepción singular fue la del gentilhombre flamenco Jehan Lhermite (1), que se detuvo en el modo en que los distintos ámbitos del conjunto monacal eran utilizados al final del reinado de Felipe II, prestando una atención muy particular a los estanques.

Análogamente, algunos estudiosos contemporáneos se han interesado por el entorno y por el agua en sus diferentes manifestaciones, como Gregorio de Andrés (2) (3), Cervera Vera (4) (5) (6), Martín Gómez (7) (8), Cervera, Añón y García Frías (9) o Navascués (10). Desde un punto de vista más divulgativo, destacan dos recientes monografías sobre los Bosques Reales (11) y sobre la cerca (12).
Esta deliberada falta de consideración de los procesos de transformación del soporte geográfico en el entorno del Monasterio ha conducido a enfoques parciales y sesgados que han aislado la arquitectura de la vida cotidiana, convertida en una abstracción en la que el abastecimiento de agua o de bastimentos, la molienda, la evacuación de residuos o la manufactura de ciertos productos como el papel -la vida cotidiana, en definitiva-, no han tenido cabida.

Por ello, la novedad y relevancia del presente estudio radica precisamente en que aborda tales procesos de transformación a partir de la construcción de las distintas e imprescindibles infraestructuras dentro del Parque Real que rodeaba el Monasterio; pero también reside en la oportunidad y características de sus usos y funcionamiento, pues aparecen las bodegas, lagares, estanques, jardines, fuentes, arcas, viñas, olivares y molinos que citaba fray José de Sigüenza (13), «que todo esto se hizo para el servicio de la casa, tanto como se plantaba de árboles frutales y arboledas» (Figura 1).

La superposición de todos estos elementos en el espacio geográfico ha ido construyendo no sólo el territorio, sino los paisajes que se han sucedido hasta la actualidad y que han sido reflejados tanto en la cartografía, los dibujos históricos o los vuelos, como en los documentos escritos.

El ámbito temporal se extiende desde la fundación del Monasterio hasta principios del siglo XX, cuando se desarrollaron los nuevos proyectos de urbanización en los municipios ya independientes de San Lorenzo y de El Escorial de Abajo.

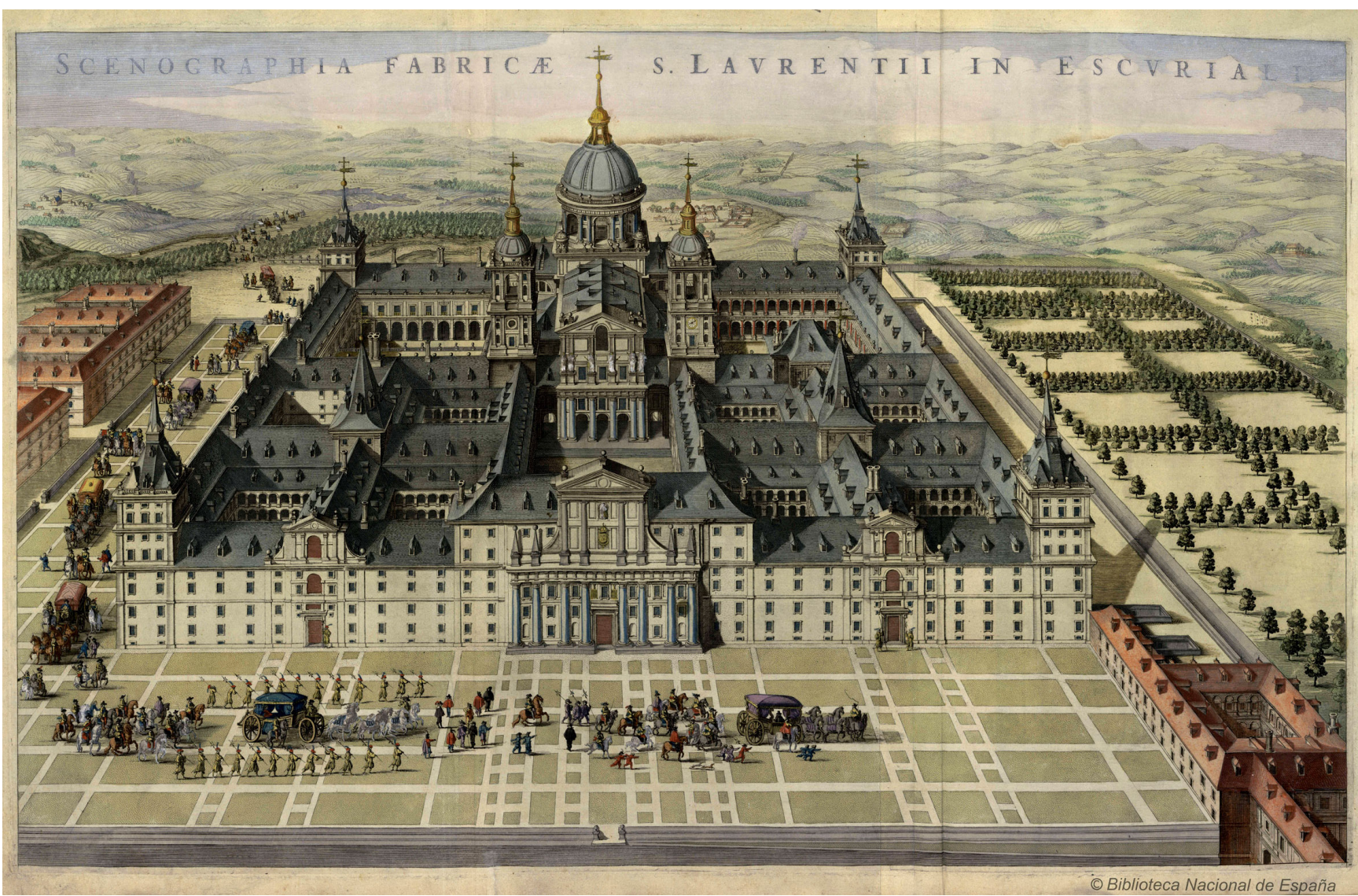

Figura 1. Atlas Blaeu, 1667: Scenographia Fabricae S. Lavrentii in Escuriali (detalle). Biblioteca Nacional de España, Madrid. El fondo de esta variante del 'Séptimo Diseño' de Herrera y Perret muestra los principales hitos del Parque Real; el molino está representado junto al margen derecho. 


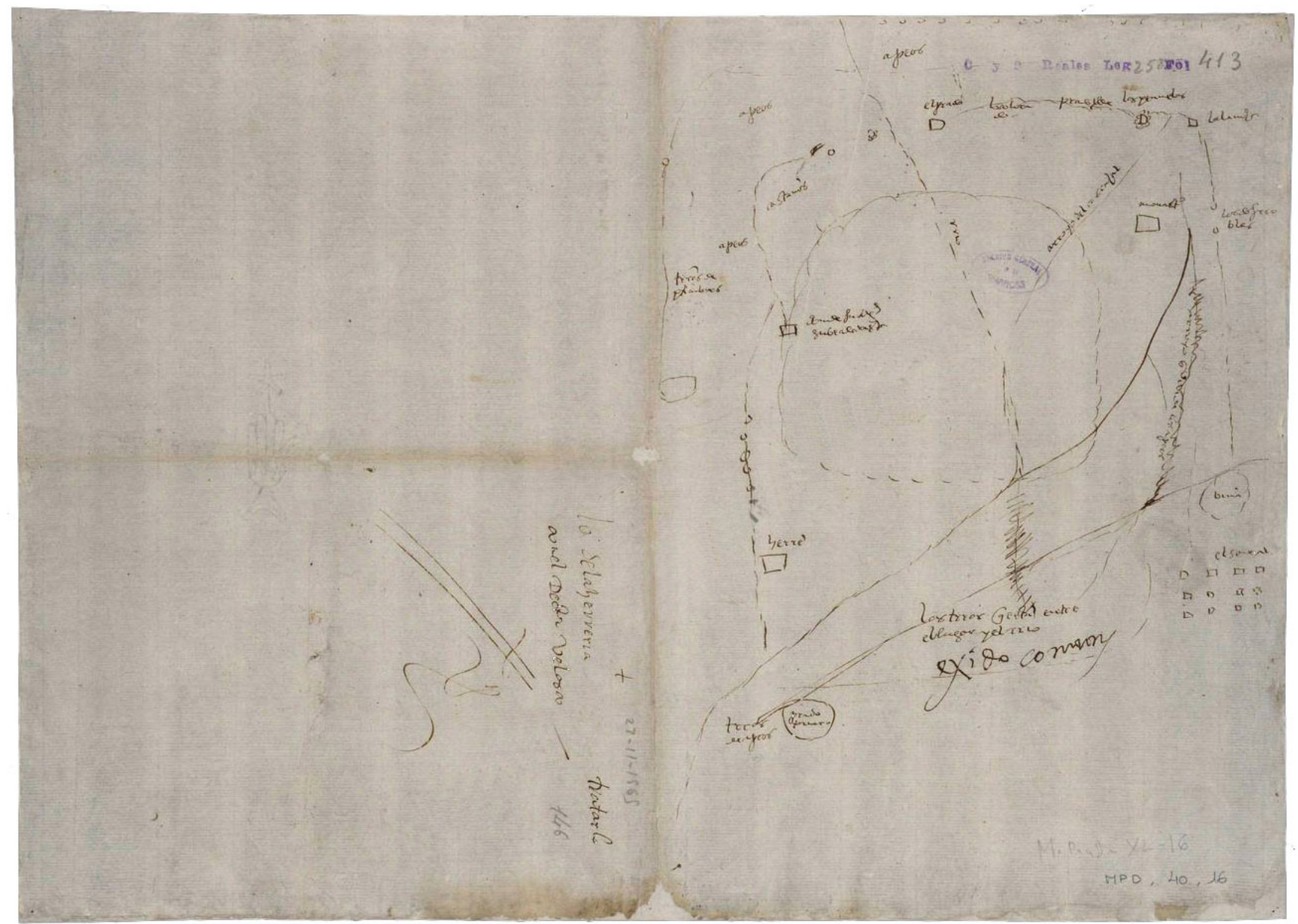

Figura 2. Anónimo, 1565: Traza muy sencilla de la dehesa de la Herrería para proceder a su ordenación y sirva de mayor ornato a los alrededores del Monasterio. Ministerio de Cultura, Archivo General de Simancas, Valladolid. Se aprecian tanto el río como el arroyo del Cerbunal, procedente de la fuente homónima, en cuyo curso medio se construiría la presa del Romeral. El monasterio está representado como un rectángulo en el ángulo superior derecho.

\section{EL MARCO GEOGRÁFICO}

La acotación espacial de la investigación se circunscribe, pues, a los territorios que constituyeron las fincas más próximas al servicio del Monasterio, esto es, a los comprendidos entre el arroyo del Tercio por el este y las sierras del Sistema Central por el oeste, y desde Guadarrama y Alpedrete por el norte hasta la Dehesa de Fuente Lámparas, al sur de las Machotas. Comprende tanto las cumbres serranas -con altitudes que oscilan entre los $1.745 \mathrm{~m}$ de Abantos y los 1.558 m del Puerto de Malagón- y sus escarpadas laderas, como el piedemonte con sus vastas zonas boscosas y adehesadas. Esta zona de la rampa está constituida por berrocales y jalonada de cerros, entre los que destaca la Atalaya Real (926 m de altitud). En ella se han conformado pequeñas depresiones o navas, zonas semihúmedas en las que se han depositado las arenas procedentes de la erosión del granito. La escasa pendiente provoca que el avenamiento de los cauces sea difícil y que se formen numerosas charcas y humedales como las lagunas de Las Radas, del Chicharrón y del Castrejón, entre otras, y en muchas de las cuales se han construido pequeños diques para su aprovechamiento ganadero, pesquero y cinegético.

Por este ámbito discurren los cursos altos del río Guadarrama y de su afluente el Aulencia; el primero lo hace por la parte más oriental de la zona de estudio, y a él vierten los dos arro- yos Guateles -frecuentemente citados en la correspondencia de Felipe II-, que atraviesan las fincas de el Campillo y Monesterio y en los que se instalaron diversos ingenios, así como el arroyo Loco -así llamado por su cauce variable.

El curso alto del Aulencia se denomina arroyo del Batán, de significativo topónimo, que atraviesa La Herrería y el resto de las fincas de oeste a este hasta confluir con el arroyo del Tercio -que constituye el límite geográfico del Parque Real por el este.

De entre los afluentes del Aulencia también merecen citarse, por su proximidad a la fábrica del Monasterio, el arroyo del Romeral -que desciende por la ladera sur de Abantos- y el del Arca del Helechal -que lo hace por la Solana del Ventisquero-. Como más adelante se verá, los tres resultaron idóneos para la construcción de presas.

Son también numerosos los manantiales y las fuentes.

\section{EL ABASTECIMIENTO DEL CONJUNTO}

Siguiendo la doctrina vitrubiana, en la elección de la ubicación del Monasterio, la existencia de fuentes que asegurasen el abastecimiento de agua en todas las estaciones del año resultó una cuestión prioritaria, así como la de arroyos con caudal suficiente para construir presas y molinos (Figura 2). 


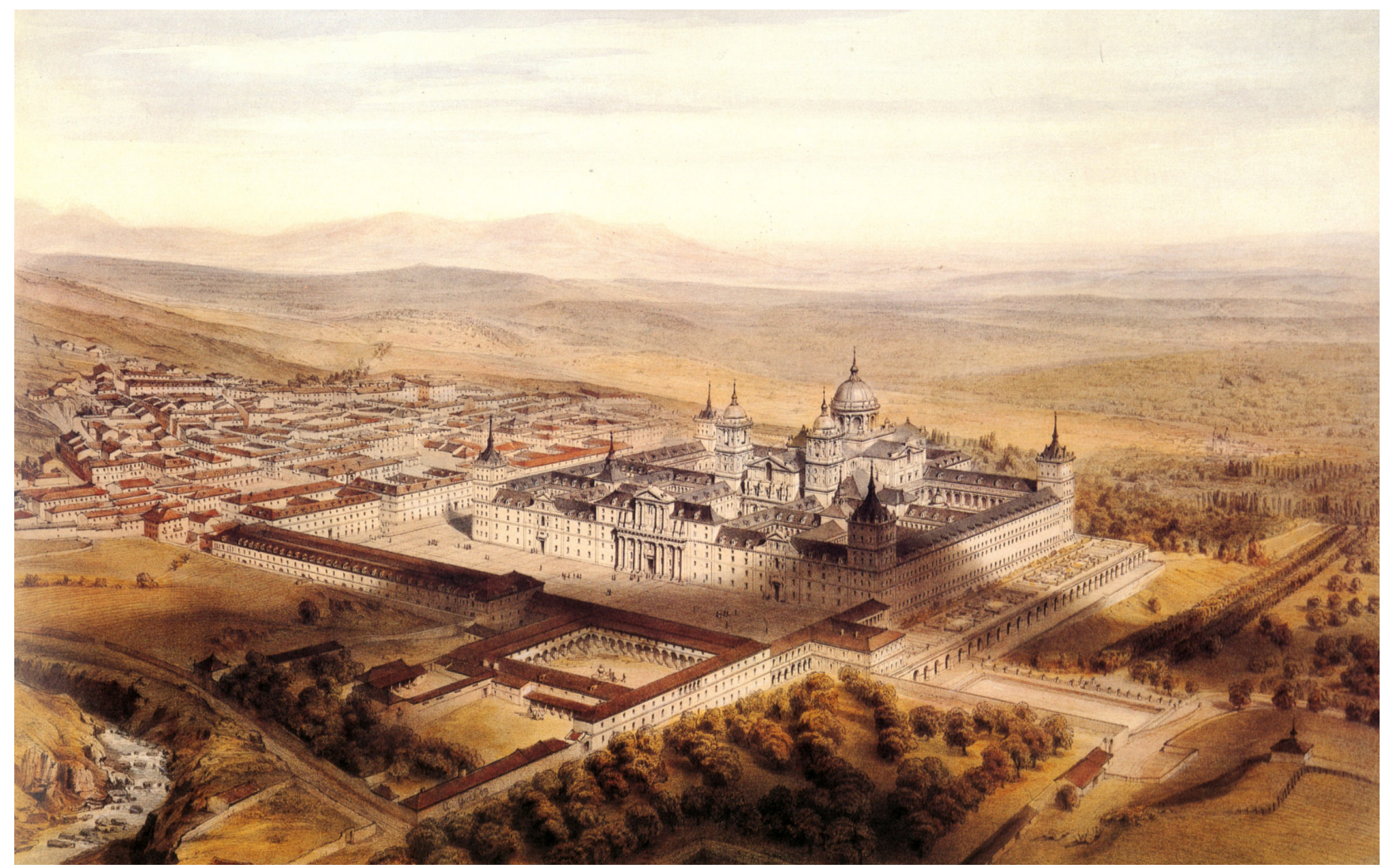

Figura 3. Alfred Guesdon, ca. 1855: St. Laurent de L'Escurial: vue prise de la route du Palais en haut. Biblioteca Nacional de España, Madrid. Abajo a la izquierda se aprecia el arroyo del Romeral, así como la derivación a la Compaña y al molino. A la derecha, el Estanque.

De hecho, «el sitio de la fuente de Blasco Sancho» ya aparece citado en una carta del secretario de Felipe II, Pedro del Hoyo, fechada el 3 de diciembre de 1561 IVDJ caja 82, envío 61, $\mathrm{n}^{\circ}$ 11\}; y también la no menos caudalosa de Matalasfuentes -renombrada luego como fuente de la Reina-, «más apartada hacia el Poniente» y que tenía «alguna mas sal» (14).

El aprovechamiento de fuentes y arroyos para el abastecimiento del conjunto monacal aparece descrito en un manuscrito fechado en 1645 (15), que Gregorio de Andrés (2) atribuye al P. Nicolás de Madrid.

De acuerdo con las descripciones antiguas, con el reconocimiento de Enrique Repullés en 1899, con las Hojas kilométricas 1:2.000 del Catastro de Rústica de 1860-1870, y tras efectuar el imprescindible trabajo de campo, hemos reconstruido el trazado desde el arroyo del Cascajal -La Barranca en los apeos del siglo XVIII-, en el que la captación se realiza en una primera arca de sillería a la que también afluyen por un encañado de barro las aguas procedentes del arca del San Juan sobre el arroyo del Tobar (Figura 3).

Este arca del Cascajal, se encuentra en el curso medio del arroyo homónimo; en ella «se hizo un arco por encima del arroyo, y entra [el agua] siete u ocho pies en alto y cae en el arca. Esta agua se tiene por mejor que la del arroyo y sustenta el mes de agosto y septiembre ella sola [...] Metida el agua en los caños desde la misma arca viene orilla del camino, de arquilla en arquilla, que hay de una en otra cien pies, poco más o menos» (15). En la última «antes de llegar a las casas y talleres» y al pósito - probablemente el lugar que mantiene el topónimo de los Pilares- había una derivación a La Compaña, mientras el caño principal seguía hasta el arca de los Repartimientos, en la actual Plaza de Benavente. De esta última arca partían los ocho caños que abastecían al Monasterio atravesando entre las dos primeras Casas de Oficios y la Lonja en dirección a la fachada norte, donde penetraba en el edificio; y junto a ella había un estanque «donde cogen agua los vecinos», hoy convertido en fuente. Toda la red en el interior de los edificios, y en particular del monasterio, fue construido siguiendo los criterios de máxima eficiencia y accesibilidad, con un complejo sistema de llaves que no sólo permitió hasta el siglo XX que el agua llegase con presión a todo el edificio hasta una altura de 33 pies, sino que los diferentes ramales se pudieran reparar sin perjudicar el funcionamiento del resto.

La derivación a La Compaña atravesaba el camino para entrar en el Plantel, girando después a levante para entrar «por debajo de la carpintería, donde se sacó un caño al corral primero de la gallinería, e hízose fuente a la manera de pozo [...] y caminando el agua por la dicha carpintería fue a dar a un arca, que está en el corral de la leña del horno [...] y de ahí va a dar agua a la cocina de la Compaña; y tomando allí lo que ha menester con todo el remanente va a dar agua a una bóveda que está para el servicio de la zapatería, y tomando allí la que ha menester [...] revuelve a mano derecha por un conducto y va al estanque de la huerta» (16) (Figura 4).

Por otra parte, desde que el rey Carlos III consiguió en 1767 que la Comunidad Jerónima consintiera en construir en los terrenos que hoy ocupa el pueblo de San Lorenzo y se promulgaron las primeras Ordenanzas, la población comenzó a crecer y con ella la demanda de agua. Para paliarla se encargó al arquitecto mayor Juan de Villanueva entre 1770 y 1780 la construcción de las presas del Romeral y del Infante. 


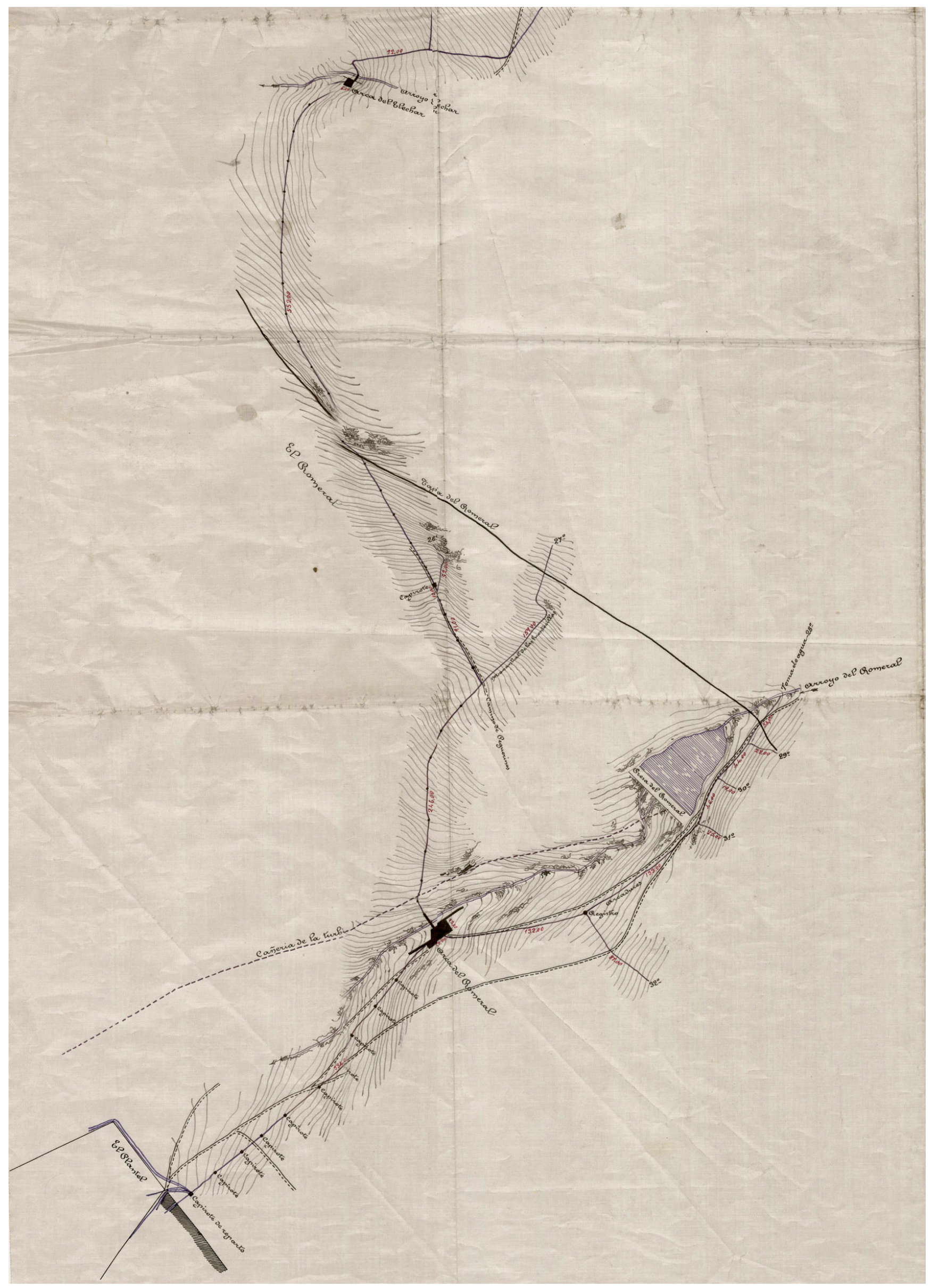

Figura 4. Enrique Repullés,1899: Viaje de aguas del Real Sitio de San Lorenzo de El Escorial (detalle). Patrimonio Nacional, Archivo del Palacio Real, Madrid. 
En 1899 aún se mantenía intacto el encañado original, pero a raíz del crecimiento de la población de San Lorenzo el sistema de abastecimiento del conjunto hubo de ser revisado por el arquitecto Enrique Repullés. Éste levantó todo el trazado junto al sobrestante Mariano Pardo, y sustituyó los encañados de barro originales por tuberías de plomo con mayor sección, reduciendo las pérdidas y las averías \{APR planos 2272, 2325-2330 .

Poco después, en 1908 el ingeniero Rafael Ripollés realizó la sustitución de las tuberías en el Viaje Grande; estas mejoras afectaron especialmente al arca del Romeral y a la presa homónima \{APR planos 1871-1873\}. Su capacidad se aumentó entre 1921 y 1931 con la construcción de la Presa Nueva del Romeral; también se realizó una nueva traída de aguas subterránea desde el embalse del Tobar.

Como contrapartida, al remodelarse la plaza de Benavente en 1911 se demolió el Arca de los Repartimientos, de la que la fuente actual guarda memoria.

\section{EL AGUA, EL RECREO Y EL RIEGO: LOS ESTANQUES}

Junto al edificio de la Botica y el muro de los nichos se construyó el estanque grande de la huerta. Diseñado por Francisco de Mora, éste debió terminar sus trazas antes de 1588 , pues el 2 de junio de ese año se contrató la ejecución de la obra a los maestros de cantería y albañilería Pedro del Carpio y Alonso de Torres, como «destajeros y laborantes en la fábrica del monasterio de San Lorenzo el Real» (17).

Las condiciones técnicas y económicas fueron redactadas por Gonzalo Ramírez, veedor y contador de la fábrica monacal, y por Fray Antonio de Villacastín. En ellas se estipulaba que la obra habría de hacerse en sillería engrapada, además de la escalera de bajada a la huerta y una o dos escaleras más para bajar al vaso, y con un andén todo en derredor \{Archivo Municipal de El Escorial, Protocolo de Francisco Escudero\}.

Las obras se debieron concluir con rapidez pues el padre Sepúlveda afirma que se acabó «de poner en la perfección que tiene» en mayo de 1589. Con el agua se regaba la huerta y se mantenía una piscifactoría, pues el rey mandó traer tencas, carpas y otras especies de Flandes y Milán (18).

En La Fresneda el rey también quiso construir un conjunto de estanques que, además de servir a los usos citados, permitieran un uso lúdico. Ya en mayo de 1563 se cita en una carta del prior Huete al secretario Hoyo que había enviado «al Pelegrin, tornáronme a decir que será bien que viniese el Holandés». A lo que el rey anotó al margen: «Pésame de la calentura, despachad luego por el Holandés que será bien reparar aquello, y de que le he dicho lo que le parece de ello y le volvamos a entender de lo de allá y a este propósito me escribís avisando de cómo os fuere y será bueno dar prisa a Juan Bautista» \{AGS OyB, Escorial leg. 72\}. El holandés era Petri Janson, quien ya había trabajado en otras obras reales ayudado por Pedro de Tolosa y supervisado por Juan Bautista de Toledo (Figura 5).

En julio de 1563 Janson recorrió la finca localizando los lugares adecuados para situarlos, y finalmente se construyeron cuatro, que Sigüenza (19) describió detalladamente, Lhermite (1:268) se refirió también a ese uso lúdico al recordar «que en esta estación estaban helados y sobre ellos nos deslizamos rodando sobre nuestros patines de Holanda».
Según Quevedo (20), en los estanques de La Fresneda «estaban prevenidas góndolas perfectamente construidas» por el marino Carlos de Ravasquier, y en particular un bergantín de 22 pies de largo, notable no sólo por su esmerada construcción, sino tan ricamente empavesado y alhajado, en particular la cámara de popa, que llamaba la atención de cuantos lo veían. En 1564 lo vio y usó don Juan de Austria quien dijo «Que no tenía inconveniente en pasar con él el golfo de León».

De las fuentes parece que se ocupó el milanés Juan Andrea (21).

\section{LOS DESAGÜES}

Gran parte del agua sobrante del interior del Monasterio y de las pluviales iba a los grandes aljibes que se construyeron en diferentes patios del interior de la fábrica, y eran reaprovechadas para las fuentes en un alarde de optimización de los recursos hídricos. Los sobrantes y las aguas negras eran conducidos a diversos puntos del exterior por galerías abovedadas visitables «que puede un hombre caminar enhiesto por ella» (22). Además hay que añadir los manantiales que surgen en diversos puntos del interior de la fábrica, cuyas aguas hubieron de ser también evacuadas.

Aunque en 1910 se realizó una primera revisión del saneamiento del conjunto, fue el arquitecto Anselmo Arenillas el que actualizó toda la red en 1954 \{APR, planos 1507-1508, 2260 y 3593-3607\}. La mayoría de las galerías continúa siendo accesible y visitable, y además de la ya citada procedente de La Compaña, las principales que vertían originalmente al exterior son las siguientes.

Dentro de la iglesia nace un manantial diez o doce pies bajo el suelo «junto a la esquina [del pilar] que mira a la capilla de $\mathrm{S}$. Mauricio»; se construyó un pozo en piedra y el agua se encañó bajo la nave «y se juntó con otros manaderos que hay entre el altar de Nuestra Señora y el de S. Pedro, y el pilar que está frontero de ellos, y volviendo a mano derecha se metió en la madre principal, que va por debajo de las gradas del altar mayor»y, obviamente, se dirige al Panteón. Este caño es el que causó tantos problemas para terminar esta parte del Monasterio, puesto que al retomarse las obras en 1643 y bajar el suelo cinco pies más de lo proyectado el Panteón comenzó a inundarse. De hecho, hasta que fray Nicolás de Madrid no dio con el problema y desvió las aguas no se pudo concluir la obra y trasladar los cuerpos reales, lo que sucedió finalmente el 17 de marzo de 1654, casi cincuenta y seis años después de la muerte de Felipe II.

Este conducto general o «desaguadero» del Bosquecillo recibía también el agua de los estanquillos del Patio de los Evangelistas, de los patinillos de las Campanas y del patio de Mascarones, además de las aguas interiores de la parte oriental del edificio (23) (Figura 6).

Desde la balsa que hay junto a la torre de las Damas -la noreste-, sale el agua por un conducto bajo el jardín de Levante y entre las dos escaleras «y camina derecho al Escorial por debajo de tierra, hasta salir [...] fuera de la pared del dicho Bosquecillo, como sesenta pies más o menos de la puerta que llaman de Carpio, que está al rincón y mira al Escorial» (24).

Finalmente, el desaguadero que sale al Jaral por debajo de la puerta condenada de la huerta acababa en la fuente de Lobregón; recogía «las aguas de las cocinas, balsas del convento, colegio, seminario y enfermería del convento y balsa que está 


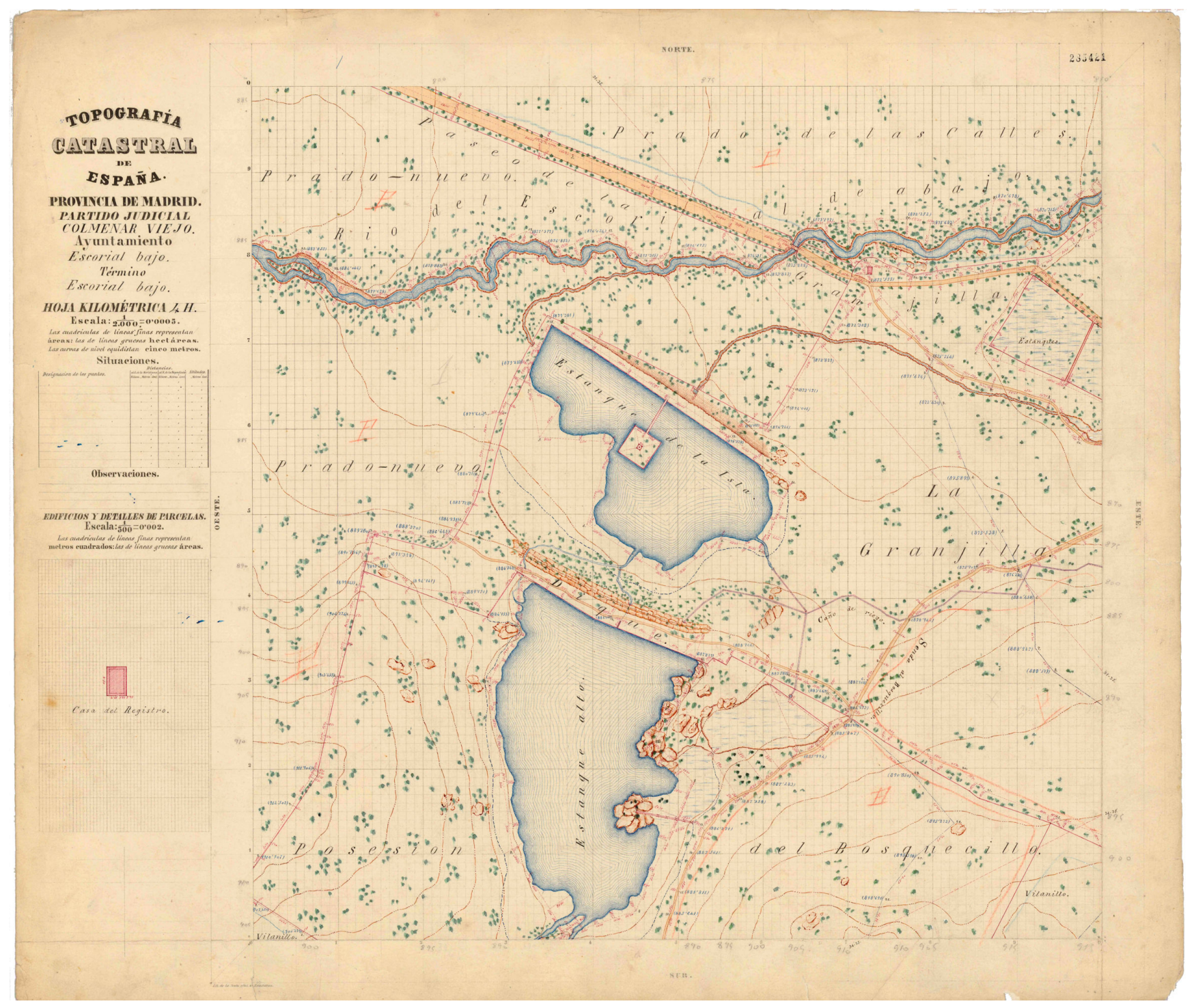

Figura 5. Topografía Catastral de España, Catastro de rústica, 1860-1870. Término Escorial Bajo, Hoja kilométrica 4-H. Instituto Geográfico Nacional, Madrid. La hoja representa los estanques de La Fresneda.

debajo de la botica, del patio principal que se dice de los reyes del pórtico, de toda la lonja [...] y se despeña por entre la viña y dicho estanque [de la huerta]» (25).

\section{EL AGUA Y LA SALUD: LOS POZOS DE NIEVE}

Desde la Antigüedad se consideraba que la nieve tenía propiedades medicinales por su capacidad de refrescar bebidas y conservar alimentos, y a ello se dedicaron varios tratados. El método seguido para transformar la nieve en hielo consistía en cortar la nieve con palas en primavera, para trasladarla después a los pozos a lomos de caballerías dentro de capazos impermeabilizados con hojas. Una vez en el pozo, los pisoneros la disponían en tongadas de entre 20 y $50 \mathrm{~cm}$ de espesor separadas por capas de paja o helecho, y la prensaban hasta que se transformaba en hielo. En verano se cortaban los bloques de hielo y se transportaban durante la noche hasta el Monasterio, de nuevo a lomos de caballerías.

Aunque existía un pozo en la zona de la Pizarra -El Campillo- anterior a la fundación del Monasterio, para servicio del conjunto escurialense se construyeron un total de cuatro pozos más: tres de ellos en las cumbres de San Juan, situados en zonas de umbría por encima de los $1.600 \mathrm{~m}$ de altitud -Navazo de la Pulga, Cuelgamuros-, a los que se accedía por la Vereda de los Pozos. En 1594 se pagaba una primera nómina de los peones que habían trabajado en la cuadrilla del sobrestante Gregorio Reglero \{AME XII, 31\}, y en 1595 ya estaba en funcionamiento el de San Juan de Malagón, pues se pagó a Diego Alonso de Mirantes, nevero de la casa de la Fuenfría, por dieciocho días que asistió en encerrar la nieve en el pozo, y por veintinueve haces de paja centenaza para ir poniendo entre la nieve $\{$ AME XIII, 12\}.

Consisten en un cono invertido de directriz circular de mampostería, de unos $7 \mathrm{~m}$ de diámetro en la boca y con una profundidad en torno a los $12 \mathrm{~m}$. Aunque hoy sólo se conserva uno de ellos entero -pues estuvo en funcionamiento hasta 1934-, permite apreciar que originalmente se protegían con una construcción también de mampostería, de planta rectangular y cubierta abovedada sobre el pozo y con cerchas de madera en el resto, que estaba protegida con teja a dos aguas. El cerramiento se reforzaba en los lados mayores con potentes contrafuertes para soportar la presión del hielo, mientras en la parte inferior existían varios huecos para la carga y la descarga, que se realizaba con el auxilio de poleas. 


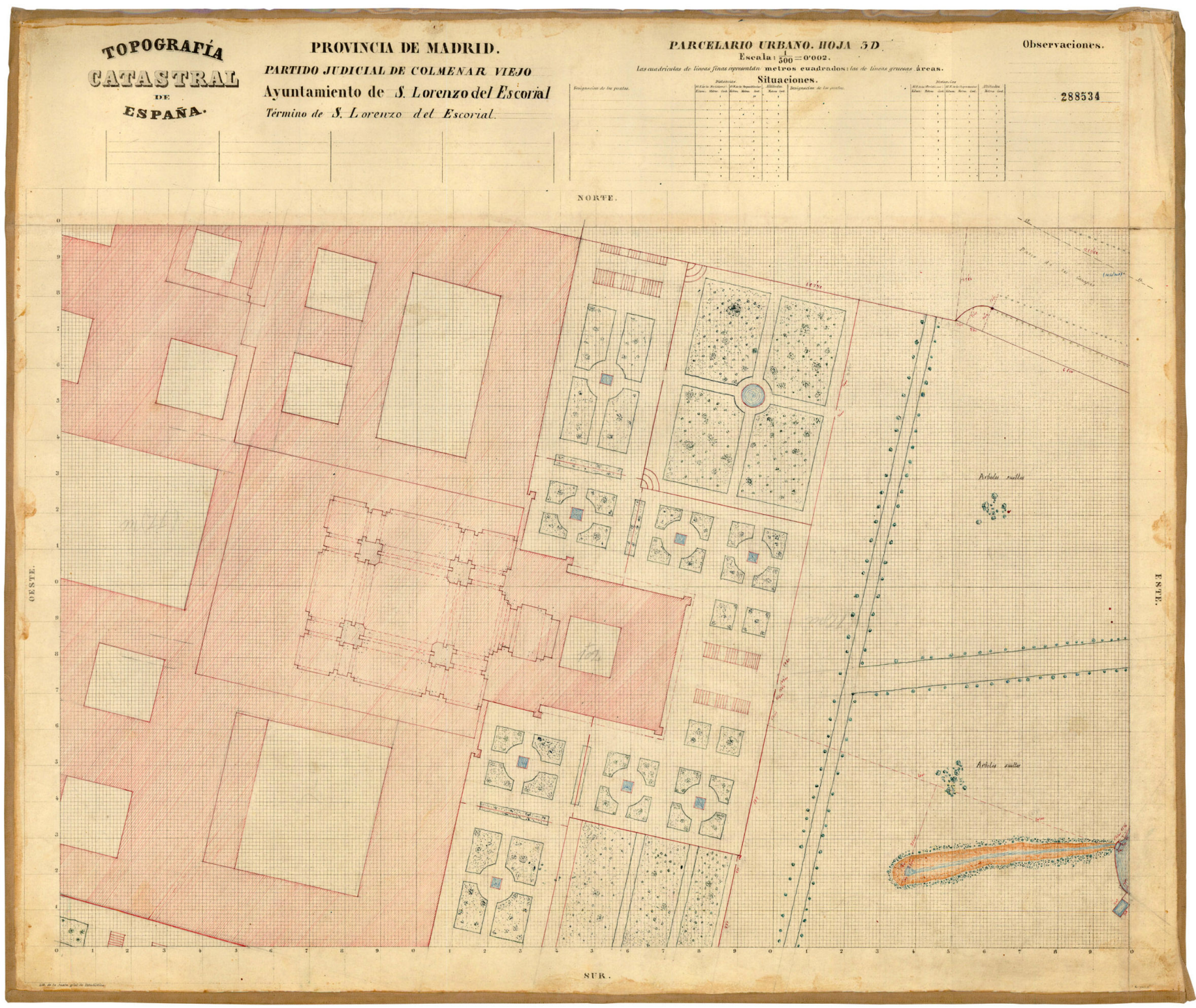

Figura 6. Topografía Catastral, Parcelario urbano, 1860-1870. Ayuntamiento de S. Lorenzo de El Escorial. Hoja 3-D. Instituto Geográfico Nacional, Madrid. En la parte inferior derecha está representada la salida del desagüadero y el estanque del Bosquecillo, sustituido recientemente por una piscina.

El fondo está ligeramente inclinado hacia el desagüe por una alcantarilla para que el agua procedente del deshielo pueda escurrir sin derretir el resto (Figura 7).

En el Capítulo celebrado en diciembre de 1609 los jerónimos decidieron construir otro pozo «en lo alto de Campillo, en las vertientes que llaman de Cuelgamuros [...] para las granjas de verano, para los enfermos y huéspedes principales, y para el Convento algunos días de los caniculares y de gran calor en el estío», pero también para vender la nieve.

Finalmente, el pozo que se encuentra junto al Monasterio se utilizaba para almacenar el hielo procedente de los neveros de la montaña.

Construido por la Comunidad, probablemente con las trazas de Francisco de Mora \{IVDJ, envío 21, fol. 173\}, actualmente está muy restaurado.

\section{LOS MOLINOS}

Los molinos hidráulicos se construyeron en la zona con propósitos diferentes, como la fabricación de papel, la molienda de cereal, el corte de piedras duras para el monasterio, e incluso la fabricación de armas.

Se distribuyeron sobre tres cursos diferentes: el arroyo del Batán -el río Aulencia en su curso alto-, el río Guadarrama y su afluente el arroyo Guatel $1^{\circ}$.

Las primeras menciones a estos ingenios aparecen en dos informes. El primero se refería a los estanques, y fue remitido en 1566 por el contador de la fábrica Andrés de Almaguer al secretario del rey, Pedro del Hoyo: «Todos convienen en que se puede hacer molino en el estanque y batán y se ahorrará mucho gasto» $\{$ AGS OyB, Escorial leg. 6\}.

El segundo informe es el Memorial sobre algunas cosas que se podrían hacer en la dehesa de la Herrería, para que estuviese mucho más agradable y con mucho más lustre \{AGS leg. 2, fol 48\}. En él se aprecia la necesidad de construir un molino harinero en el entorno del Monasterio, pues «en el Escorial y en la Fresneda y en otros lugarejos cerca de ellas, hay falta de moliendas, especialmente en los años secos que van a moler a unos molinos de Guadarrama, y en ellos hay poco agua y tanta prisa a moler que, algunas veces, quien lle- 
va una carga de trigo para que se muela, espera tres o cuatro días».

En consecuencia, se planteó la construcción de un primer molino harinero en la salida de la Herrería, y más tarde, un segundo en el interior del conjunto de la Compaña.

El Manual de la Hacienda de este Monasterio de San Lorenzo el Real, y de sus rentas y granjerías elaborado en 1605 y renovado en 1652 \{APR Patronatos, leg.154\} menciona que «en esta dehesa [de la Herrería] se hicieron en tiempo del mismo fundador una [es]clusa y presa de piedra, que todo está junto en el río Abulencia (sic), poco antes del Prado Tornero, y esto dicen que sirvió para serrar allí jaspes y mármoles en la fundación de esta casa, y acabada la obra se dedicó todo a molino de papel y últimamente a molino harinero que le he conocido yo moler muchísimos años, hasta que en el año pasado de [1]643 le destecharon, y no han quedado más que las paredes, la presa, aunque no muy bien compuesta, y el cubo para el agua que bajaba de los rodetes, que eran dos piedras; este cubo está bueno y dicen que es alhaja»; después «mandó su majestad fundar y hacer de nuevo el molino de pan de dos ruedas que ahora posee esta casa y arrienda, que está en el arroyo de la Herrería, del cual se sirvió este Monasterio para su gasto muchos años, hasta que se labró en la Compaña el que ahora tenemos; y antiguamente había junto a este molino en la casa que allí hay grande, cierto ingenio para aserrar las piedras de jaspe que se asentaron en el altar mayor de este convento; y después mandó su Majestad fundar allí junto el molino de papel que ya está caído, por haberse experimentado que su costa era más que el provecho».

Su construcción empezó en 1576, cuando se fijaron las condiciones "para hacer las paredes de la casa del molino» AME $\mathrm{V}, 18$ \}, pero hasta 1578 no se contrató al albañil Gaspar Ruiz para levantarlas. Ese mismo año Antón Lozano se obligaba

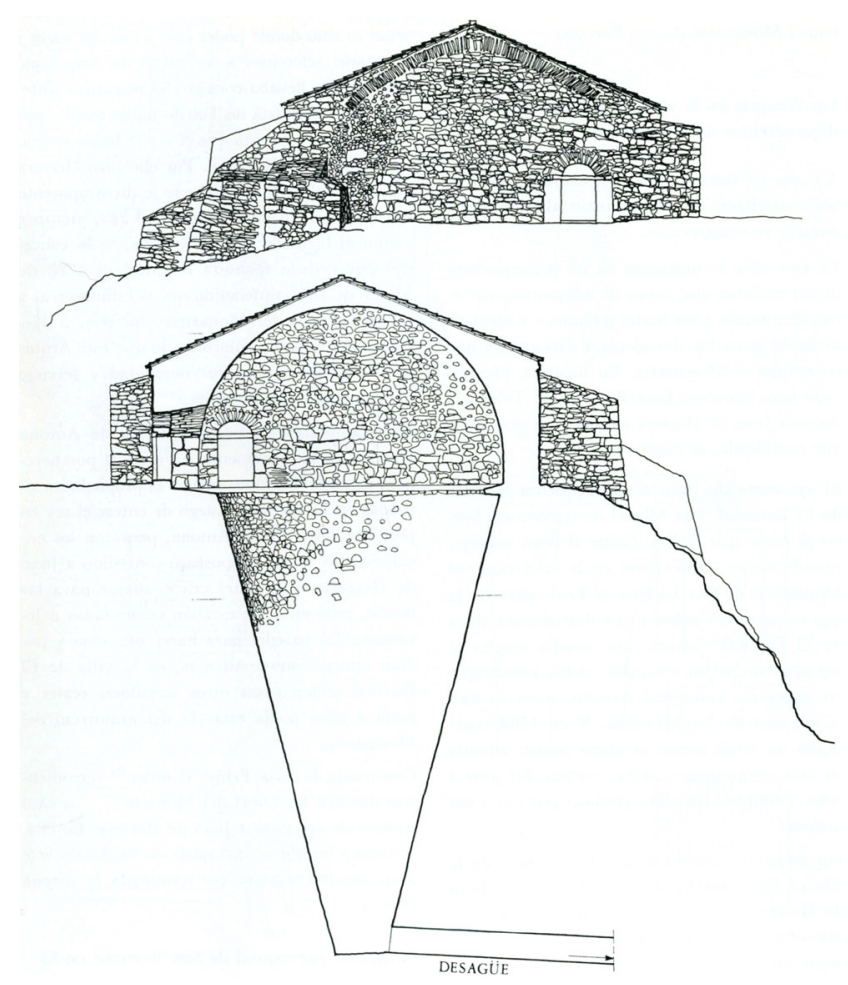

Figura 7. Luis Cervera Vera, 1986: Alzado y sección de un pozo de nieve. a labrar y traer desde Hoyo seis piedras redondas harineras para las ruedas del molino \{AME VI, 23\}. Un año antes, en 1577 el cantero Nicolás de Rivero se obligaba a hacer la presa y el cubo \{AME VI, 9\}.

El molino del jaspe o «molino caído» se levantó para labrar la piedra de mármol destinada al retablo de la Basílica; y en 1579 Francisco Campero, maestro de cantería, se obligaba «a la obra de cantería para el ingenio de labrar, bruñir y aderezar la piedra de mármol» \{AME VI, 42\}, pero fue finalmente ejecutada por los canteros Francisco Campero y Alonso Esteban \{AME VII, 14\}, y tasada en 1580; la froga fue concertada con Juan Montoya ese mismo año \{AME VII, 25\}.

Respecto al interior, el italiano Pedro de la Mola y el carpintero Andrés de Herrera se comprometieron en 1579 «a hacer un ingenio conforme a la traza y modelo de Jácome de Trezo y a satisfacción de Juan de Herrera» \{AME VII, 5\}. Cuatro años después Mola seguía asistiendo como oficial «en la obra modelo e ingenio del molino de jaspe» \{AGS, Memoriales de partes, leg. 4\}. Paulo Morigi (26) dijo de este molino que contaba con la tecnología más avanzada de su tiempo, y que los jaspes se cortaban «con el artificio del agua que hacía trabajar todo el hierro a golpe de cuatro martillos».

Desde 1584 se sucedieron las compras de sierras tanto para el molino de jaspe como para el taller, encargadas todas ellas al herrero Alonso de Llanos \{AME IX, 4 y 22$\}$.

Por último, el molino de pan tenía dos ruedas y se arrendaba, «porque para el servicio de esta casa basta el molino de pan que hay dentro de la Compaña» $\{\mathrm{AME} X, 2\}$.

Por último, el Manual de Hacienda elaborado en 1605 \{BME, 187.II.14\} describe que el molino de pan estaba junto al de papel, y que éste era entonces «de ningún provecho».

Para su adaptación a molino de papel, en 1590 estaba trabajando el entallador Pedro Mola \{AME XI, 39 y XII, 7\} y en 1592 se pagó a Julián Martínez por la obra de carpintería \{AME XI, 45\}. Ese mismo año Rodrigo de Holanda pintó de ocre y verde montaña todas las rejas, puertas y ventanas «de los molinos del ingenio y del papel» \{AME XII, 8 y 16\}.

Estuvo funcionando como molino harinero hasta mediados del siglo XVIII.

Al describir la Compaña, Lhermite (27) dice de su molino harinero: «se levantó allí un molino de agua de muy rara y extraña invención que no costó menos de 80.000 escudos y que sólo muele durante tres o cuatro meses al año, en concreto en los meses de invierno, cuando el agua llega hasta allí desde las montañas. Pero como esta agua fluye en muy pequeñas cantidades y con bastante escasez, se ha inventado para que este molino pueda moler una ingeniosa industria que consiste en un gran receptáculo desde donde esta agua, después de atravesar un canal con grandísima furia, cae desde lo alto sobre las ruedas del molino. Éste, impulsado por esta sola fuerza, se pone en movimiento y consigue moler».

Este ingenio se construyó en el lienzo norte del edificio, entre el corral de la leña de la cocina y el de la gallinería. Se trata de un molino de cubo con dos ruedas y tahona que fue diseñado por Francisco de Mora; la obra de cantería fue realizada por Pedro del Carpio y Yuste González, y la del caz lo fue por 
el cantero Miguel Sánchez y los albañiles Esteban Frontino y Gaspar Rodríguez, todo ello en 1596 \{AME XII-9 y XIII-16\}. Los trabajos de carpintería corrieron a cargo de Juan García, y se midieron y tasaron en 1597 \{AME XIV, 4 y 7$\}$. Ese mismo año Alonso Sánchez Zerudo sacó y desbastó las piedras del molino \{AME XIV, 5\} y de la tahona $\{$ AME XIV, 2\}, lo que demuestra que aún estaba en construcción (Figura 8).

En el cárcavo del molino se halló un manantial que se cubrió con una bóveda hasta pasar la esquina de la huerta y atravesar la cerca de la Herrería, donde, ya descubierto, confluía en el arroyo homónimo.

Aún se conserva bajo la cocina el cárcavo, el canal de entrada del agua y la cuba cilíndrica.

El molino del Batán se construyó también en el arroyo homónimo, aguas arriba de los anteriores ingenios y «pegado al prado que llaman de Matacuadrado [...] como un cuarto de legua de casa camino de Robledo». Las condiciones de la obra del «batán y lavatorio de la ropa de la casa» se redactaron en 1582 \{AME VIII, 4\}, y se contrataron con Juan Romero, maestro de albañilería, y con Juan de la Obarrieta, vecino de Pastrana $\{$ AME VIII, 2\}. En 1584 se encargaba al calderero abulense Gaspar de Arroyo «una caldera de cobre con dos caños para el batán» \{AME IX, 4\}. Finalmente, la obra de carpintería se encargó ese mismo año a Antonio Rodríguez \{AME IX, 8\}.
Según el Manual de Hacienda, «no se sabe si era riguroso batán para batanar paños, o sólo para lavar la ropa de lana de los Religiosos». En 1605 se conservaba el edificio, pero no quedaba rastro de la maquinaria y sólo se utilizaba como lavadero.

Siempre según el Libro de Actas Capitulares del Monasterio, se autorizó que se hiciera una prueba para la fabricación de paños de lana blancos treintenos con la lana de la cabaña del Monasterio, y tras el buen resultado obtenido, en 1780 se aprobó acometer la obra para que «se componga y ponga corriente el batán de la comunidad para que batanen en él los paños de la nueva fábrica». No resultó tan rentable, pero sí una fuente de gastos y de ruido que funcionó hasta el segundo cuarto del siglo XIX.

Casi un siglo después, en el anuncio publicado en el Boletín General de Ventas de Bienes Nacionales de 27 de junio de 1870 figuran «las ruinas de un edificio que fue batán», añadiéndose que el comprador habría de respetar «la servidumbre de aguas [...] con inclusión del estanque o presa titulada del Batán y demás obras propias de la conducción de aguas». Recientemente fue reconstruido para servir de restaurante, aunque no se respetaron ni el secadero, ni los pilones ni la alberca.

Por último, en la finca de El Campillo existía «una costosísima fábrica donde se labraban toda clase de armas, que recibió el nombre de molino de las armas», probablemente construido

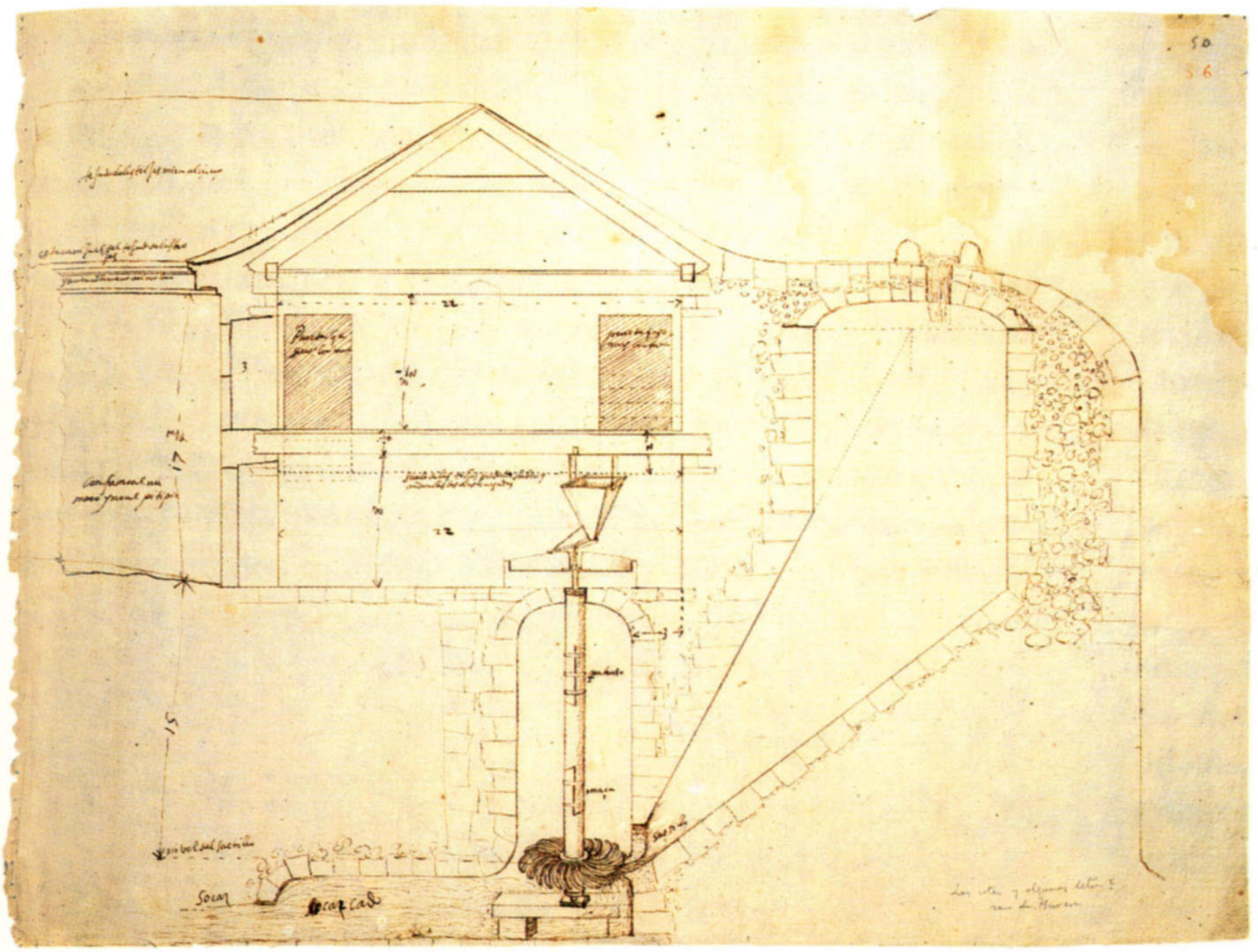

Figura 8. Atribuido a Francisco de Mora, ca. 1590: Sección del molino de La Compaña. (Lám. XXXVII, Catálogo López Serrano). Patrimonio Nacional, Biblioteca del Palacio Real, Madrid. 
por los Maqueda, que fueron los propietarios anteriores (28). También conocido como Molino del Jaral, actualmente se encuentra en ruinas, muy próximo al $\mathrm{km} 12,600$ de la carretera Guadarrama-San Lorenzo sobre el arroyo Guatel $1^{\circ}$.

Muy cerca, en la vecina finca de Monesterio se construyó, por una decisión del Capítulo de 1626, un molino de papel sobre el río Guadarrama que era necesario para la impresión de los libros del Nuevo Rezado y de diversas Bulas cuyo monopolio tenía el Monasterio. Estuvo en funcionamiento hasta 1678, año en que se cerró porque al parecer hacía mucho ruido y espantaba la caza. Siempre al servicio del Monasterio, se trasladó a Arenas de San Pedro.

Este molino, también muy arruinado, es el que aparece representado en la hoja kilométrica 15D del Catastro de Rústica realizado en $1860-1870$.

\section{CONCLUSIONES}

La previsión y el buen oficio de los constructores del conjunto de fincas y edificios del Monasterio, han hecho posible que una gran parte de las infraestructuras hidráulicas hayan llegado hasta el siglo XX, muchas de ellas en funcionamiento, $\mathrm{y}$ que incluso las ruinas de las fábricas sean tan sólidas que aún permiten su reconocimiento y estudio.

Como se ha podido apreciar, en esta investigación no sólo ha tenido un gran peso la documentación escrita, pues el trabajo de campo ha resultado imprescindible en la comprobación no sólo de las localizaciones precisas de todos los elementos o hitos territoriales, sino que ha permitido realizar un levantamiento cuidadoso de cada uno. Y en esta labor, la documentación gráfica y cartográfica histórica -lo que incluye vistas y fotografías antiguas-, junto con los vuelos realizados en diferentes años, no sólo es muy abundante, sino que constituye una fuente de gran interés y complementaria a los textos (29).

La combinación de estas tres fuentes ha permitido descubrir las trazas de los ingenios que poblaron el territorio, las huellas de las diferentes culturas, de los hitos históricos y de la vida cotidiana en el conjunto escurialense, que han conformado los sucesivos paisajes hasta la actualidad.

\section{REFERENCIAS}

(1) Lhermite, J. (2005). El Pasatiempos. Aranjuez: Doce Calles.

(2) Andrés, G. (1965). Descripción de la fontanería del Monasterio de El Escorial hecha en 1645. En Zarco, J. (Ed.) Documentos para la historia del Monasterio de San Lorenzo el Real de El Escorial, Vol. 8 (pp. 219-318). Madrid: Imprenta Helénica.

(3) Andrés, G. (1975). Toponimia e historia de la Montaña Escurialense. Anales del Instituto de Estudios Madrileños, (11): $15-26$.

(4) Cervera-Vera, L. (1985a). El conjunto monacal y cortesano de La Fresneda en El Escorial. Boletín de la Real Academia de Bellas Artes de San Fernando, 60:149-196.

(5) Cervera-Vera, L. (1985b). Juan de Herrera diseña el puente sobre el río Guadarrama. Anales del Instituto de Estudios Madrileños, 22:55-79.

(6) Cervera-Vera, L. (1986). Conjuntos y caminos en torno al Monasterio de San Lorenzo el Real. En Población y Monasterio [El Entorno], IV Centenario del Monasterio de El Escorial (pp. 37-64). Madrid: Ministerio de Obras Públicas.

(7) Martín-Gómez, P. (1986a). La evolución y los sistemas en la obra de El Escorial. En Fábricas y orden constructivo [La Construcción], IV Centenario del Monasterio de El Escorial (pp. 83-96). Madrid: Ministerio de Obras Públicas.

(8) Martín-Gómez, P. (1986b). La fundación y orígenes de la población del Real Sitio de San Lorenzo. Población y Monasterio [El Entorno], IV Centenario del Monasterio de El Escorial (pp. 15-35). Madrid: Ministerio de Obras Públicas.

(9) Cervera, L., Añón, C., García-Frías, C. (2003). La Fresneda: un lugar de Felipe II en el entorno de El Escorial. Doce Calles.

(10) Navascués, P. (1985). Puentes de acceso a El Escorial. Archivo Español de Arte, 58(230):97-107.

(11) Ramírez-Altozano, J.J. (2009). Historia de los Bosques Reales de San Lorenzo del Escorial. Madrid: Visión.

(12) Sánchez-Meco, G., Rosado, V. (2007). La cerca histórica de los bosques del Real Sitio de San Lorenzo de El Escorial. San Lorenzo de El Escorial: Sociedad de Fomento y Reconstrucción del Real Coliseo Carlos III.

(13) Sigüenza, J. (1605). La Fundación del Monasterio de El Escorial, (vol. III Historia del orden de San Gerónimo). Madrid: Turner (Reed. 1986).

(14) Sigüenza, J. (1605). La Fundación del Monasterio de El Escorial, (vol. III Historia del orden de San Gerónimo), p. 21. Madrid: Turner (Reed. 1986).

(15) Archivo del Monasterio de San Lorenzo de El Escorial. Noticias de todas las fuentes que tiene este Rl. Monasterio de Sn. Lorenzo, Caja XV, fol. 3 r.

(16) Archivo del Monasterio de San Lorenzo de El Escorial. Noticias de todas las fuentes que tiene este Rl. Monasterio de Sn. Lorenzo, Caja XV, fol. 15v-16r.

(17) Íñiguez-Almech, F. (1965). Las Trazas del Monasterio de San Lorenzo de El Escorial, pp.62-63. Madrid: Real Academia de Bellas Artes de San Fernando.

(18) Cabrera-de Cordova, L. (1619). Filipe Segundo Rey de España, p.925. Madrid: Luis Sanchez.

(19) Sigüenza, J. (1605). La Fundación del Monasterio de El Escorial, (vol. III Historia del orden de San Gerónimo), pp. 398-399. Madrid: Turner (Reed. 1986).

(20) Quevedo, J. (1848). Historia del Real Monasterio de San Lorenzo, p.147. Madrid.

(21) Chías, P. (2013). Territorio y paisaje en el entorno del Monasterio de San Lorenzo de El Escorial: planos y vistas desde el dibujo de Hatfield House a Guesdon. Revista EGA, (22):38-49, doi: http://dx.doi.org/10.4995/ega.2013.1687. 
(22) Archivo del Monasterio de San Lorenzo de El Escorial. Noticias de todas las fuentes que tiene este Rl. Monasterio de Sn. Lorenzo, Caja XV, fol. 19r.

(23) Chías, P., Abad, T. (2013). Terrestrial and Aerial Ground-penetrating Radar in use for the Architectural Researches: Ancient 16th Century Water Supply and Drainage at the Monastery of El Escorial (Madrid, Spain). ISPRS International Archives of the Photogrammetry, Remote Sensing and Spatial Information Sciences, XL-5/W2:177-182, doi: http:// dx.doi.org/10.5194/isprsarchives-XL-5-W2-177-2013.

(24) Archivo del Monasterio de San Lorenzo de El Escorial. Noticias de todas las fuentes que tiene este Rl. Monasterio de Sn. Lorenzo, Caja XV, fol. 18r.

(25) Archivo del Palacio Real, San Lorenzo. Advertencias hechas por fray Diego de Ciudad Real y el veedor de la fábrica del Escorial sobre los conductos generales y desagüaderos de la casa de san Lorenzo, 1692, leg. 109.

(26) Morigi, P. (1593). Historia brieve dell'Augustissima casa d'Austria, con la descrittione della rara al mondo fabrica dello Scuriale di Spagna. Bergamo.

(27) Lhermite, J. (2005). El Pasatiempos, pp. 367-368. Aranjuez: Doce Calles.

(28) Cervera-Vera, L. (1986). Conjuntos y caminos en torno al Monasterio de San Lorenzo el Real. Población y Monasterio [El Entorno], IV Centenario del Monasterio de El Escorial (p. 59). Madrid: Ministerio de Obras Públicas.

(29) Chías, P. (2014). Fincas y cazaderos reales en el entorno del Monasterio de San Lorenzo de El Escorial: tradición medieval e influencia flamenca. Revista EGA, (23):46-53, doi: http://dx.doi.org/10.4995/ega.2014.2171. 